\title{
The impact of associated tenotomies on the outcome of incomplete phalangeal osteotomies for lesser toe deformities
}

\author{
Eduardo Nieto-García', Javier Ferrer-Torregrosa2², Leonor Ramírez-Andrés², Elena Nieto-González², \\ Alfonso Martinez-Nova ${ }^{3}$ and Carlos Barrios ${ }^{4}$ (D)
}

\begin{abstract}
Background: Partial or incomplete osteotomy $(\mathrm{IO})$ of the phalanx is recently described in the literature. However, the clinical outcome and the rate of complications when applied to lesser toe deformities (LTD) have been never addressed. This study aims to find out if the association of tenotomies to incomplete or partial phalanx osteotomies has a significant impact on the clinical outcomes, the occurrence of complications, and the recovery time after surgery.
\end{abstract}

Methods: A retrospective review of two cohorts of cases operated in our institution for hallux abductus valgus (HAV) and associated LTD from 2008 to 2014 was carried out. The surgical correction of both HAV and the associated LTD was always performed by minimally invasive techniques. The study included a total of 223 patients (723 IO in 556 toes). In 129 cases, the IO for LTD correction was performed without tenotomies, and in 94, the procedure was combined with flexor and/or extensor tenotomies. Patients were assessed with the American Orthopaedic Foot and Ankle Society (AOFAS) questionnaire before surgery and at 6- and 12-month follow-up.

Results: The mean preoperative AOFAS score before surgery was similar in both cohorts. At 12-month follow-up, the cohort without tenotomies showed better recovery $(95.7 \pm 2.8$ versus $92.5 \pm 6.8 ; p<0.01$ ). AOFAS scores decreased as the number of associated LTD increased $(r=-0.814 ; p<0.001)$. Cases operated on by PO + tenotomy showed a high rate of complications such as delayed union of the osteotomy $(p<0.01)$, hypertrophic callus $(p<0.01)$, phalangeal fracture at the osteotomy site $(p<0.01)$, and lack of correction $(p<0.05)$. The overall occurrence of adverse events was $38.6 \%$ in cases operated by PO + tenotomy and $13.9 \%$ in cases receiving PO alone $(p<0.0001)$. Cases operated on without tenotomy showed a shorter time to complete recovery for daily life activities (37.4 \pm 2.3 versus $43.0 \pm 1.7$ days; $p<0.01)$.

Conclusion: The performance of associated tenotomies to incomplete phalanx osteotomies provides worse clinical outcomes, higher complication rates, and longer recovery time as compared to similar forefoot surgeries without tenotomies.

Trial registration: The study was based on retrospectively registered data starting on May 24, 2008.

Keywords: Lesser toe deformities, Percutaneous osteotomies, Tenotomy, Forefoot surgery, Minimally invasive surgery, Complications

\footnotetext{
* Correspondence: javier.ferrer@ucv.es

${ }^{2}$ School of Physiotherapy and Podiatry, Valencia Catholic University, Ramiro

Maeztu 14, 46900 Torrent, Valencia, Spain

Full list of author information is available at the end of the article
}

(c) The Author(s). 2019 Open Access This article is distributed under the terms of the Creative Commons Attribution 4.0 International License (http://creativecommons.org/licenses/by/4.0/), which permits unrestricted use, distribution, and reproduction in any medium, provided you give appropriate credit to the original author(s) and the source, provide a link to the Creative Commons license, and indicate if changes were made. The Creative Commons Public Domain Dedication waiver (http://creativecommons.org/publicdomain/zero/1.0/) applies to the data made available in this article, unless otherwise stated. 


\section{Introduction}

Lesser toe deformities (LTD) are highly frequent in the general aging population and may be associated with significant morbidity [1, 2]. These deformities occur gradually, often affect multiple toes, and are regularly associated to hallux abductus valgus (HAV) [3]. The progression of the deformity is commonly related to an imbalance between the forces of the extensor and flexor tendons about the proximal or distal interphalangeal joints [4]. Most of the LTD evolve despite podiatric care and lastly require surgical treatment. A Swedish experience based on extensive data registries suggests that almost a quarter of patients undergoing forefoot surgery had also lesser toe procedures performed [5]. Arthroplasty and arthrodesis are still widely used techniques despite their functional squeals [6-10]. The impact of associated surgeries for correction of LTD in terms of complication occurrence and final recovery after HAV is still a matter of controversy.

Minimally invasive surgery (MIS) involves bony or soft tissue procedures performed through a small incision, without direct visualization on the anatomical structures [11].

MIS techniques are attractive procedures because of their advantages: reduced soft tissue damage, shorter length of surgery and hospital stay, lower post-operative pain, and reduced infection risk. The results seem similar to those obtained using the various open techniques, although the wide range of clinical conditions makes comparisons difficult $[11,12]$.

One of the MIS procedures for LTD is the partial or incomplete osteotomy (IO) of the phalanges which consists of a unicortical osteotomy retaining an intact portion of the phalanx that may act as a fulcrum allowing the closure of the osteotomy. The first described IO was applied on the proximal phalanx of the first finger (modified Akin technique) [13, 14]. Based on their behavior, the design and execution were transferred to the rest of the phalanges of the minor toes, second to fifth. Multiple IOs can be performed in the same finger with different designs since each digital deformity is different $[15,16]$.

The goal of IO is to obtain a digital realignment that permits to recover the balance between the extrinsic and intrinsic musculature without needing to perform surgical procedures on tendon structures. In this way, the interphalangeal joints could reestablish their physiological functionality. IOs are techniques especially indicated for reducible or semi-rigid deformities of the fingers [17].

The surgical technique of IO has been well described in the literature $[16,17]$. However, the clinical outcome and the rate of complications of IO applied to LTD have never been addressed. This study aims first to analyze the clinical impact of the associated surgical procedures performed at the lesser toes at the time of HAV correction. Second and more specifically, this study evaluates the clinical efficacy and safety of IO procedures for correction of second to fifth digital deformities, focusing the more frequent complications. Third, this study analyzed if the association of tenotomies to IO techniques to correct LTD has a significant impact on the clinical outcomes, the occurrence of complications, and the recovery time after surgery.

\section{Methods \\ Participants}

From 2008 to 2014, a total of 457 patients were operated on in our institution for HAV and associated LTD. Out of these, 223 patients were selected for the final study according to the following criteria related to the type of LTD: (i) reducible deformity of the proximal interphalangeal joint in any spatial plane, that is reducible hammer and claw toe; and (ii) soft tissue involvement with extensor and flexor tendon imbalance. Cases with nonreducible deformity of the proximal interphalangeal joint (rigid hammer or claw toes) and those with isolate rigid deformity of the distal interphalangeal joint (mallet toes) were excluded. Patients with previous surgeries of the lesser toes were also excluded.

The mean age of the final 223 patients with reducible hammer or claw toes was 59 years, with a predominance of women $(n=212 ; 95.1 \%)$. There were 16 (7.2\%) diabetic patients and $6(2.7 \%)$ with rheumatoid arthritis. The right foot was treated in $52.9 \%$ of cases.

The surgical procedure for correction of both HAV and the associated LTD was always performed by MIS techniques. Out of the 223 cases, 129 (57.8\%) underwent correction of the LTD by phalangeal IO without additional tenotomies, and in 94 (42.2\%), the procedure was combined with flexor and/or extensor tenotomies.

Fifteen days prior to surgery, all patients were interviewed in order to perform the preoperative tests and complete the American Orthopaedic Foot and Ankle Society (AOFAS) scale. For the purpose of this study, the lesser metatarsophalangeal-interphalangeal subscale was analyzed [18].

\section{Surgical procedure}

HAV correction was executed in all cases by performing Reverdin and Akin osteotomies following similar criteria of Biz et al. [12]. All procedures were performed under regional anesthesia in an office-based operation theater with fluoroscopic guidance.

As for the surgical treatment of LTD, the preservation of the cortical continuity at selected point is the differentiating element of IOs as compared to other types of phalangeal osteotomies. The cortical wall acts as a natural fixing device, avoiding the displacement of the fragments and preventing the segment from shortening (Fig. 1). Two particularly relevant features at the time of execution of the 
phalangeal osteotomy should be considered in this study. The first is that the bone corrections were always performed starting from proximal to distal phalanges (Fig. 2). The second is that once the skeletal segments were realigned, an intra-surgical assessment of the reducibility of the deformity and the equilibrium achieved between the agonist and antagonist muscle groups responsible for the digital movement was assessed. In some cases, the irreducibility of the deformity required to act on the soft tissue structures, that is, on the extensor and/or the flexor digital tendons. The incomplete reducibility of the toe deformity after IO was the criteria to add tenotomies in this series of cases.

\section{Postoperative protocol}

Immediately after surgery, all patients were allowed to full weight-bearing, due to the design of the osteotomies, and the external fixing quality provided by the bandages. Patients were instructed to wear a post-surgical shoe with a rigid sole. Curing of the wounds was performed on a weekly basis. At each postoperative visit, a fluoroscopic check-up was completed to objectify the closure of the osteotomy, the maintenance and stability the phalangeal osteotomies, and adequacy of the bone segment position.

Between 30 and 40 days after surgery, when the formation of fibrous callus was verified in the performed osteotomies and the clinical evolution was satisfactory, the patient was allowed to perform his or her daily activities. Follow-up outpatient visits were carried out 6 and 12 months after surgery for clinical and radiological evolution control, recording again the AOFAS scale. All the complications found such as displacement, non-union, delayed consolidation, hypertrophic callus, and no correction were registered.

\section{Statistical analysis}

This was a retrospective study, and hence, pre-study sample size calculation was not performed. Descriptive statistical tests were first analyzed. Student $t$ test, ANOVA, Mann-Whitney test, $\chi^{2}$ test, and Fisher exact test were used in the statistical analyses of the variables depending on the nature of the variables and their intention for analysis. All statistical tests were performed using SPSS version 21 (SPSS, Inc., IBM, Chicago, IL), and $p \leq .05$ was considered significant.

\section{Results}

The demographic and clinicopathological characteristics of the two cohorts are summarized in Table 1 . There were no differences among the two cohorts in age, sex distribution, laterality, and associated diseases. Of the 223 feet, 556 toes were operated (IO group, $n$ lesser toes $=338$; $\mathrm{IO}+$ tenotomy, $n$ lesser toes $=218$ ) with a total of 723 osteotomies (IO group, $n=436$; IO + tenotomy, $n=287$ ).

The mean preoperative AOFAS score before surgery was similar in both cohorts of patients with LTD $(53.5 \pm 10.9$ for IO without tenotomy versus $53.4 \pm 8.5$ in cases with associated tenotomy). Six months after surgery, cases operated with IO alone showed better AOFAS scores than those patients receiving associated tenotomy (93.6 \pm 3.4 and $89.7 \pm 7.8$ respectively; $p<0.001$ ) (Fig. 3). Twelve months after surgery, the cohort without tenotomies showed also better recovery according to AOFAS scores (95.7 \pm 2.8 for IO without tenotomy versus $92.5 \pm 6.8, p=$ $0.001)$. At 6- and 12-month follow-up, there were no statistically significant differences between the cohorts of isolated HAV and those operated on for LTD with IO without tenotomies ( $94.5 \pm 4.7$ and $95.7 \pm 2.8$ respectively).

Figure 4 shows the preoperative and the 12 -month postoperative AOFAS mean scores discriminating the number of lesser toes operated on. The preoperative impairment of the AOFAS scores was dependent of the number of toes requiring surgical correction. The higher scores (less clinical impairment) were recorded in cases operated only of one lesser toe. AOFAS scores decreased as the number of associated LTD increased $(r=-0.814 ; p<0.001)$ (Fig. 5). Except for the cases
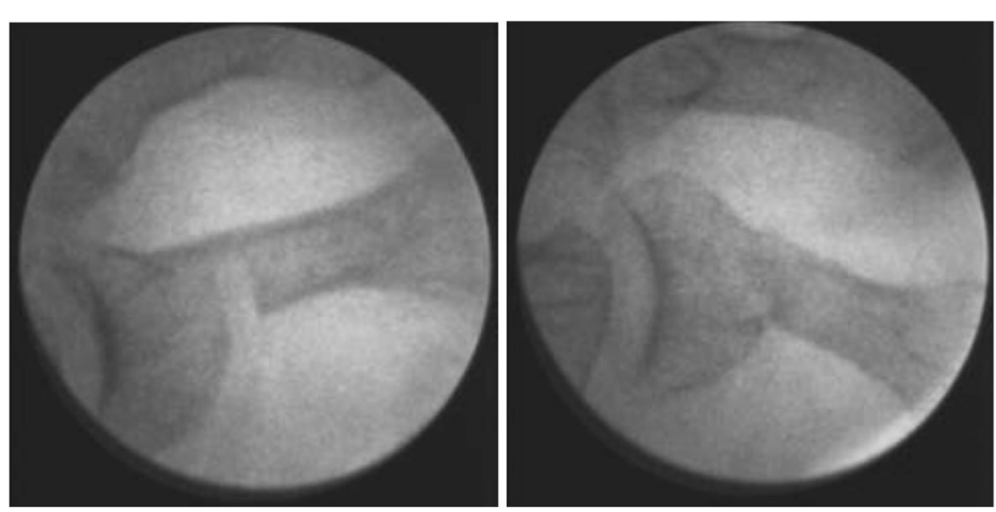

Fig. 1 Incomplete phalangeal osteotomy before and after closure 

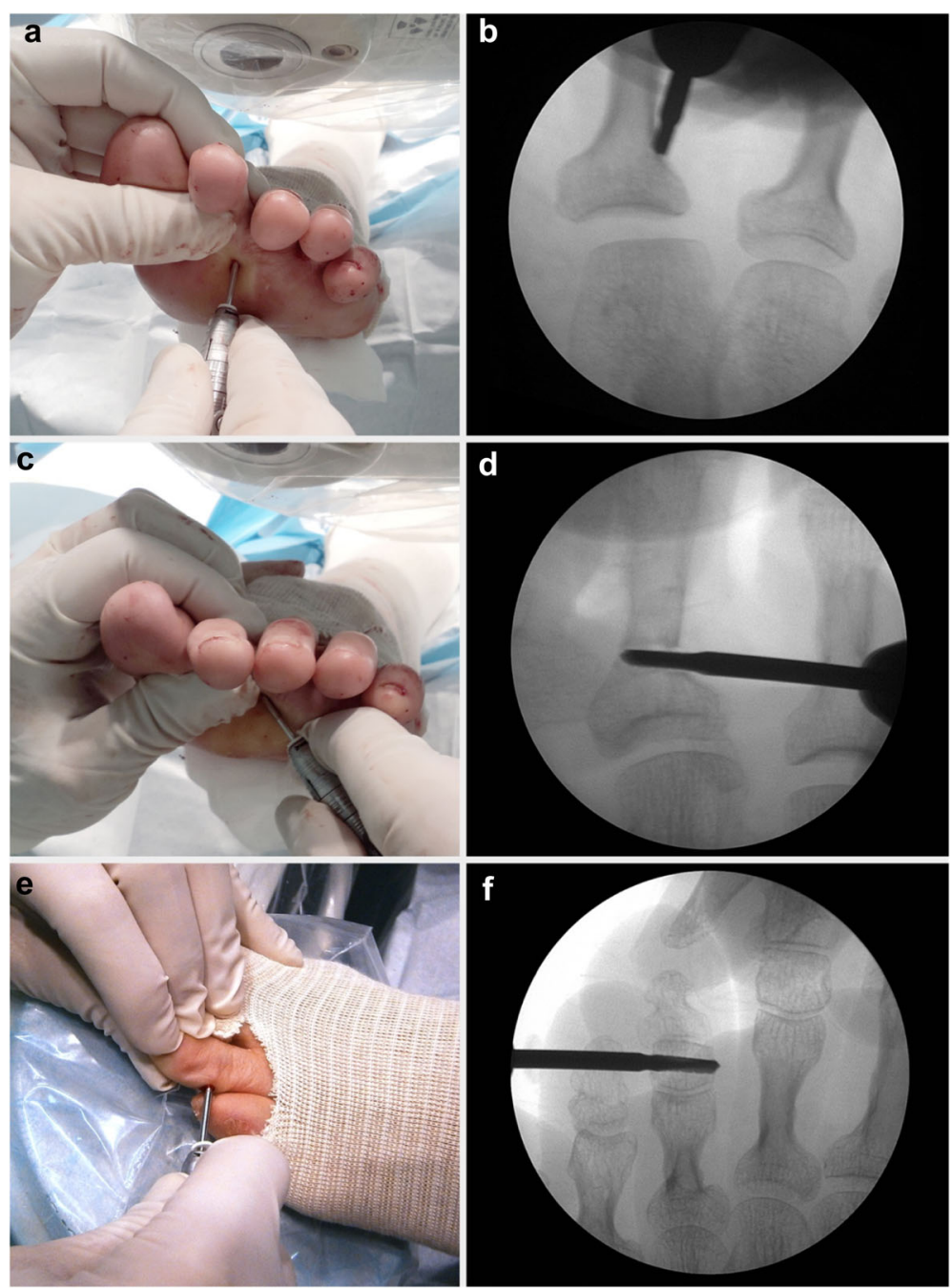

Fig. 2 Surgical planification of the incomplete phalangeal osteotomies. a Plantar approach of the proximal phalanx of the second toe. $\mathbf{b}$ Fluoroscopic view of the site of incomplete osteotomy. c Final stage of the incomplete osteotomy and $\mathbf{d}$ the fluoroscopic view. e Incomplete osteotomy at the second phalanx of the third lesser toe and $\mathbf{f}$ the fluoroscopic view

Table 1 Demographic and clinicopathological characteristics of patients

\begin{tabular}{|c|c|c|c|}
\hline Characteristics & $\mathrm{HAV}+\mathrm{IO}$ on LT $(n=129)$ & HAV + IO + tenotomy on LT $(n=94)$ & Significance \\
\hline Age (mean \pm SD) & $59.6 \pm 11.5$ & $59.5 \pm 11.3$ & $0.358^{*}$ \\
\hline \multicolumn{4}{|l|}{ Sex, $n(\%)$} \\
\hline Male & $5(3.9 \%)$ & $6(6.4 \%)$ & \multirow[t]{2}{*}{$0.533^{* *}$} \\
\hline Female & $124(96.1 \%)$ & $88(93.6 \%)$ & \\
\hline \multicolumn{4}{|l|}{ Laterality, n (\%) } \\
\hline Right & $67(51.9 \%)$ & $51(54.3 \%)$ & \multirow[t]{2}{*}{$0.786^{* *}$} \\
\hline Left & $62(48.1 \%)$ & $43(45.7 \%)$ & \\
\hline \multicolumn{4}{|l|}{ Associated disease } \\
\hline Diabetes & $6(4.6 \%)$ & $10(10.6 \%)$ & $0.115^{* *}$ \\
\hline Rheumatoid arthritis & $2(1.5 \%)$ & $4(4.2 \%)$ & $0.242^{* *}$ \\
\hline
\end{tabular}

HAV hallux abductus valgus, $I O$ incomplete osteotomy, $L T$ lesser toe

*Unpaired $t$ test

${ }^{*}$ Chi-square test 


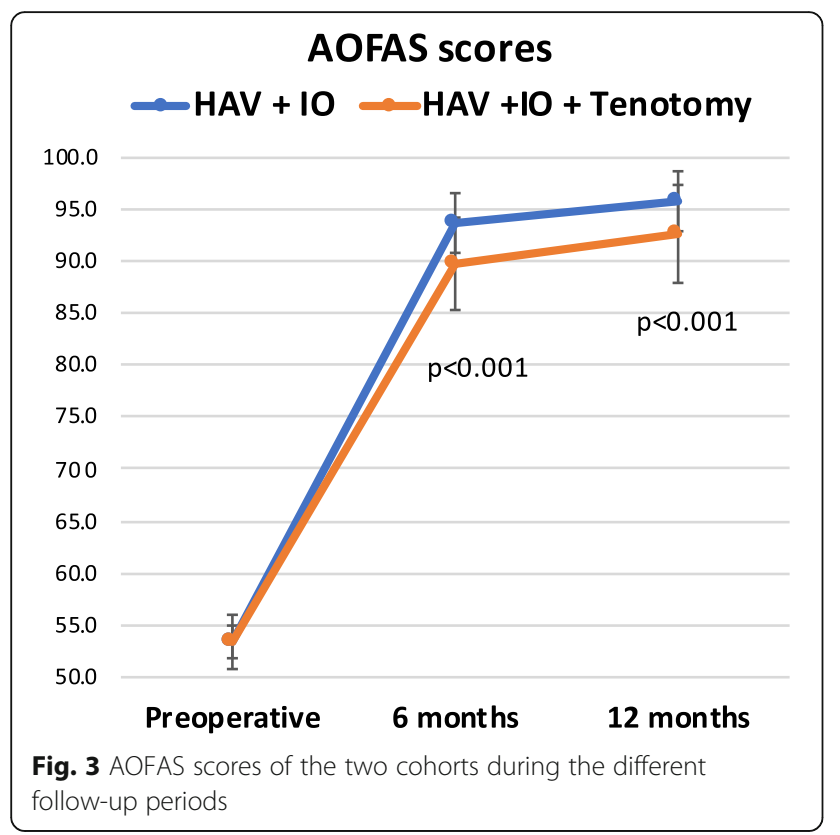

requiring correction of all five toes, the recovery was independent of the number of toes operated in association with HAV surgery.

The number of complications related to surgery and the percentage of occurrence regarding the number of operated feet and the number of procedures is summarized in Table 2. Complications of the LTD procedures were accounted separately from those occurring on the first day. When the occurrence of adverse events was considered in relation to the number of performed procedures, the complication rate of IO + tenotomy resulted

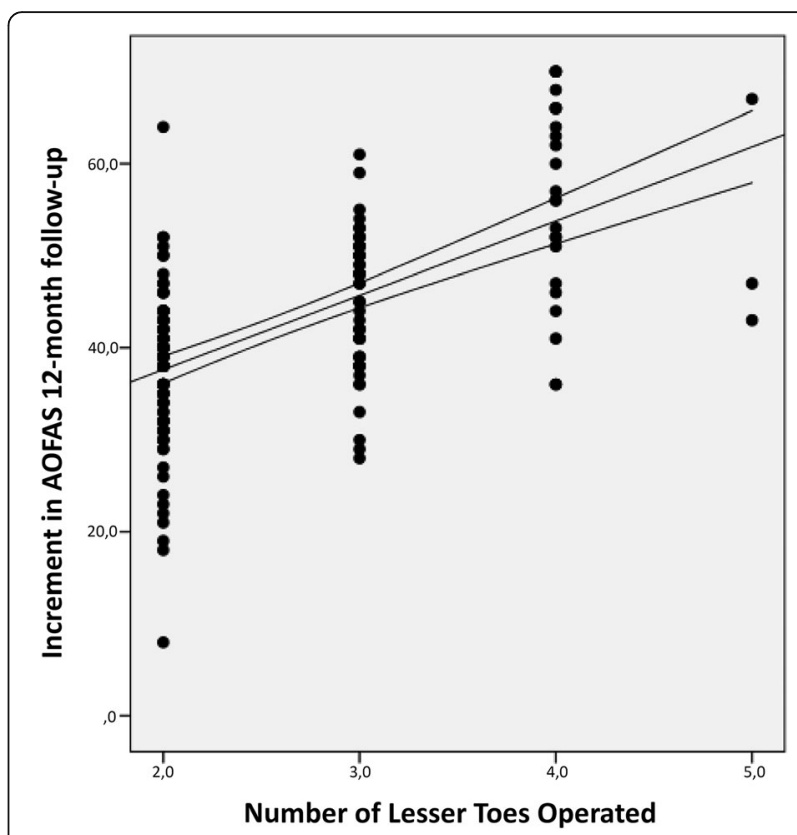

Fig. 5 Increment in AOFAS scores from preoperative period to 12month follow-up in relation to the number of lesser toes operated. The lines indicate the tendency of the mean

in $38.6 \%(111 / 287)$, while that of IO alone was $13.9 \%$ (61/436), having statistical differences that are highly significant $(p<0.0001)$. Taking all adverse events together, the complication ratio per patient was more than double in cases receiving IO + tenotomy ( 1.18 versus 0.47 ).

Cases operated on by IO + tenotomy showed a high rate of complications such as delayed union of the osteotomy $(25.5 \%$ versus $6.6 \% ; p<0.01)$, hypertrophic callus

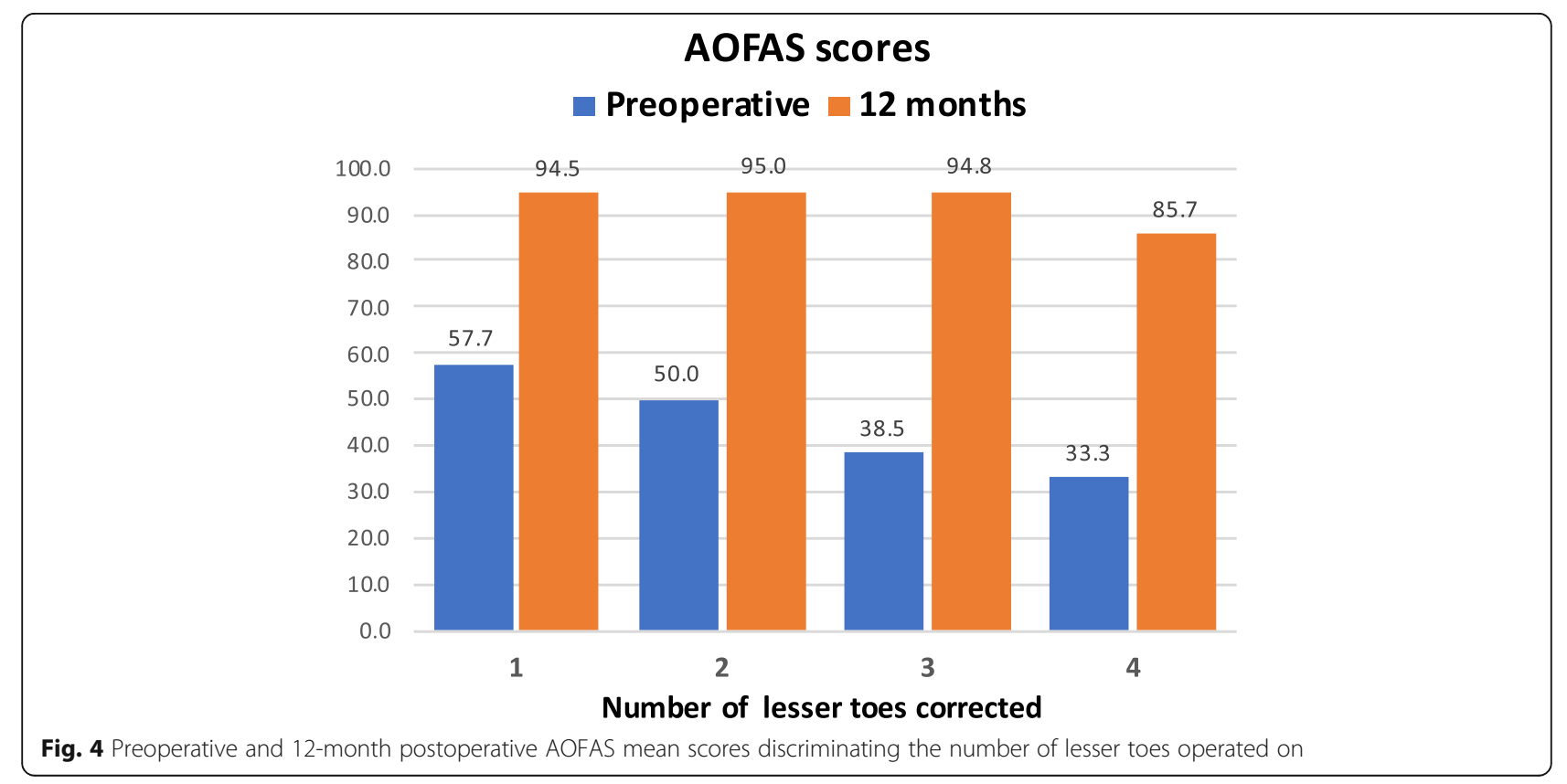


Table 2 Complications of the surgical procedures performed on the lesser toes in both cohorts

\begin{tabular}{|c|c|c|c|c|c|c|c|c|}
\hline & \multicolumn{3}{|l|}{10} & \multicolumn{3}{|c|}{$10+$ tenotomy } & \multicolumn{2}{|c|}{$\begin{array}{l}\text { Fisher's exact test } \\
\text { significance }\end{array}$} \\
\hline & $\begin{array}{l}\text { Events, } \\
n\end{array}$ & $\begin{array}{l}\text { Patients (\%), } n= \\
129\end{array}$ & $\begin{array}{l}\text { Procedures (\%), } n= \\
436\end{array}$ & $\begin{array}{l}\text { Events, } \\
n\end{array}$ & $\begin{array}{l}\text { Patients (\%), } n \\
=94\end{array}$ & $\begin{array}{l}\text { Procedures (\%), } n= \\
287\end{array}$ & Patients & Procedures \\
\hline $\begin{array}{l}\text { Intraoperative phalangeal } \\
\text { fracture }\end{array}$ & 19 & 11.4 & 3.4 & 23 & 24.5 & 8.0 & 0.082 & 0.050 \\
\hline $\begin{array}{l}\text { Postsurgical phalangeal } \\
\text { fracture }\end{array}$ & 3 & 1.8 & 0.5 & 12 & 12.8 & 4.2 & $0.002^{* *}$ & $0.002^{* *}$ \\
\hline Displacement & 14 & 8.4 & 2.5 & 20 & 21.3 & 6.9 & $0.038^{*}$ & $0.003^{* *}$ \\
\hline Delayed union & 11 & 6.6 & 1.9 & 24 & 25.5 & 8.4 & $0.000 \neq$ & $0.000 \neq$ \\
\hline Hypertrophic callus & 12 & 7.2 & 2.1 & 23 & 24.5 & 8.0 & $0.003^{* *}$ & $0.002^{* *}$ \\
\hline Non-union & 0 & - & - & 1 & 1.1 & 0.3 & 0.421 & 0.397 \\
\hline Lack of correction & 2 & 1.0 & 0.3 & 8 & 8.5 & 2.8 & $0.019^{*}$ & $0.017^{*}$ \\
\hline
\end{tabular}

10 incomplete osteotomy

${ }^{*} p<0.05 ;{ }^{* *} p<0.01 ;{ }^{\ddagger} p<0.001$

(24.5\% versus $7.2 ; p<0.001)$, postoperative phalangeal fracture at the osteotomy site $(12.8 \%$ versus $1.8 \% ; p<$ $0.01)$, displacement $(21.3 \%$ versus $8.4 \% ; p<0.05)$, and lack of correction $(8.5 \%$ versus $1.0 \% ; p<0.05)$. The only case of non-union occurred in the $\mathrm{IO}+$ tenotomy cohort. No statistically significant differences were found between the two cohorts as regards the intraoperative breakage of the cortical wall although was most frequent in the IO + tenotomy cohort. However, within the first 30 days after surgery, 3 phalanges (2.3\%) developed breakage in the group without tenotomy and 12 in the group with tenotomy $(p<0.05)$.

Figure 6 shows the risk of complications per patient in relation to the number of operated lesser toes. Differences between both cohorts were statistically significant at all stages, being complications more frequent in the tenotomy group. In each group, the percentage of osteotomies with displacement of bone fragments, delayed union, or development of a hypertrophic callus increased as the number of operated lesser toes increased (Figs. 7, 8, and 9). The increase is especially relevant when three or four lesser toes are operated.

Cases operated on without tenotomy showed a shorter time to complete recovery for daily life activities $(37.4 \pm 2.3$ versus $43.0 \pm 1.7$ days; Mann Whitney test, $Z=11.813, p<0.001)$.

\section{Discussion}

To date, there is no consensus regarding the most appropriate surgical technique for LTD. Most likely, one of the reasons could be the wide variation in definitions of the deformities and indications for treatment. In a Dutch experience, a high proportion of orthopedic departments around the country had any protocol or agreement for

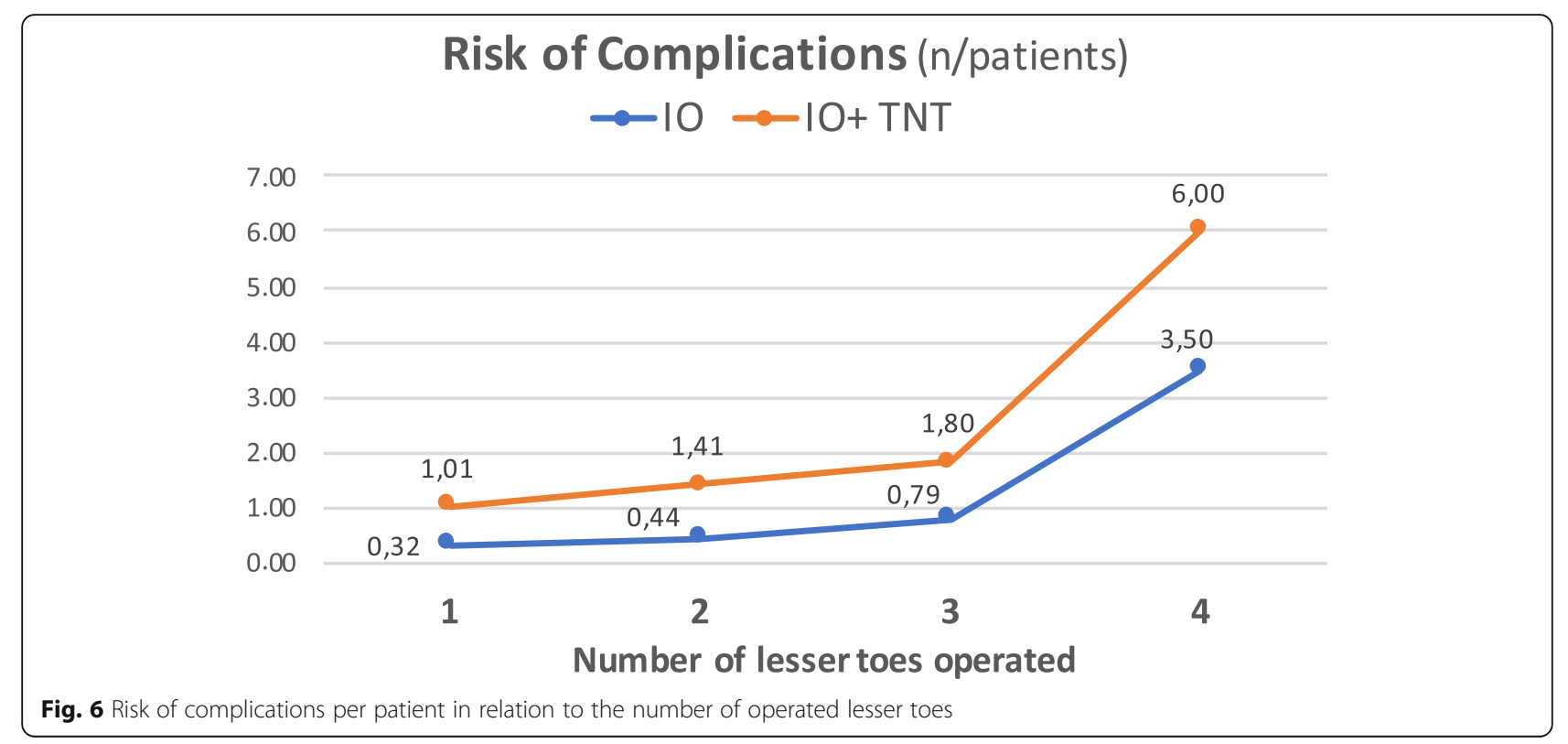




\section{Hypertrophic Callus}

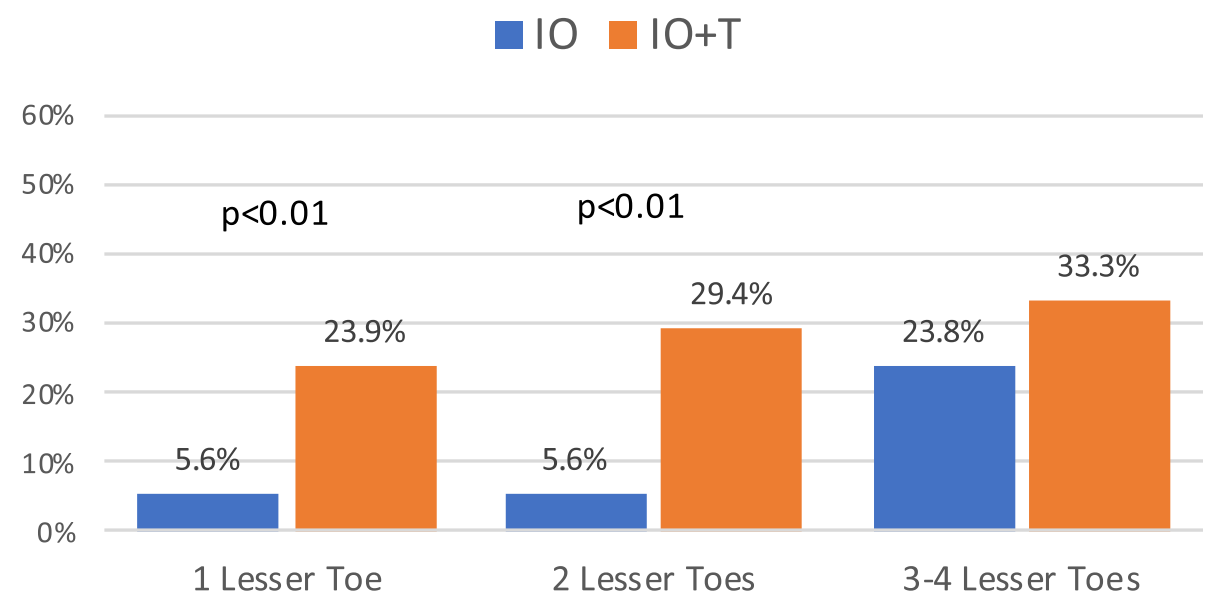

Fig. 7 Occurrence of hypertrophic callus at the site of osteotomies in relation to the number of operated lesser toes (IO: complete osteotomy; $\mathrm{T}$ : tenotomy)

the treatment of LTD [1]. This fact explained the broad spectrum in indications and performed interventions not only among the different hospitals but also within the departments.

Based on the latest evidence, several strategies for management of lesser toe deformities suggest different algorithms depending on the type of deformity [4]. These algorithms exclusively contemplate toe shortening procedures such as resection arthroplasty or arthrodesis, but never phalangeal osteotomies. For flexible mallet toes caused by a flexor digitus longus tight tendon, percutaneous flexor tenotomy is advocated to correct the deformity $[3,9]$.

The series include only cases with reducible hammer or claw toes, that is, flexible deformity of the proximal interphalangeal joint. Reducible hammer and claw toes were not considered clinically different and therefore were not analyzed separately within groups. Lesser toe deformities affecting the stiffness of the distal interphalangeal joint (rigid mallet toes) were excluded from the study because they commonly require resection arthroplasty or arthrodesis and cannot be treated by incomplete phalangeal osteotomies.

The treatments of lesser toes deformities by MIS are mostly based on soft tissue release, such as joint capsulotomies and tenotomies. The procedures on the bone depend on the residual deformities, the reducibility, and the toe length $[11,19,20]$. Some authors use complete osteotomies for the correction of digital

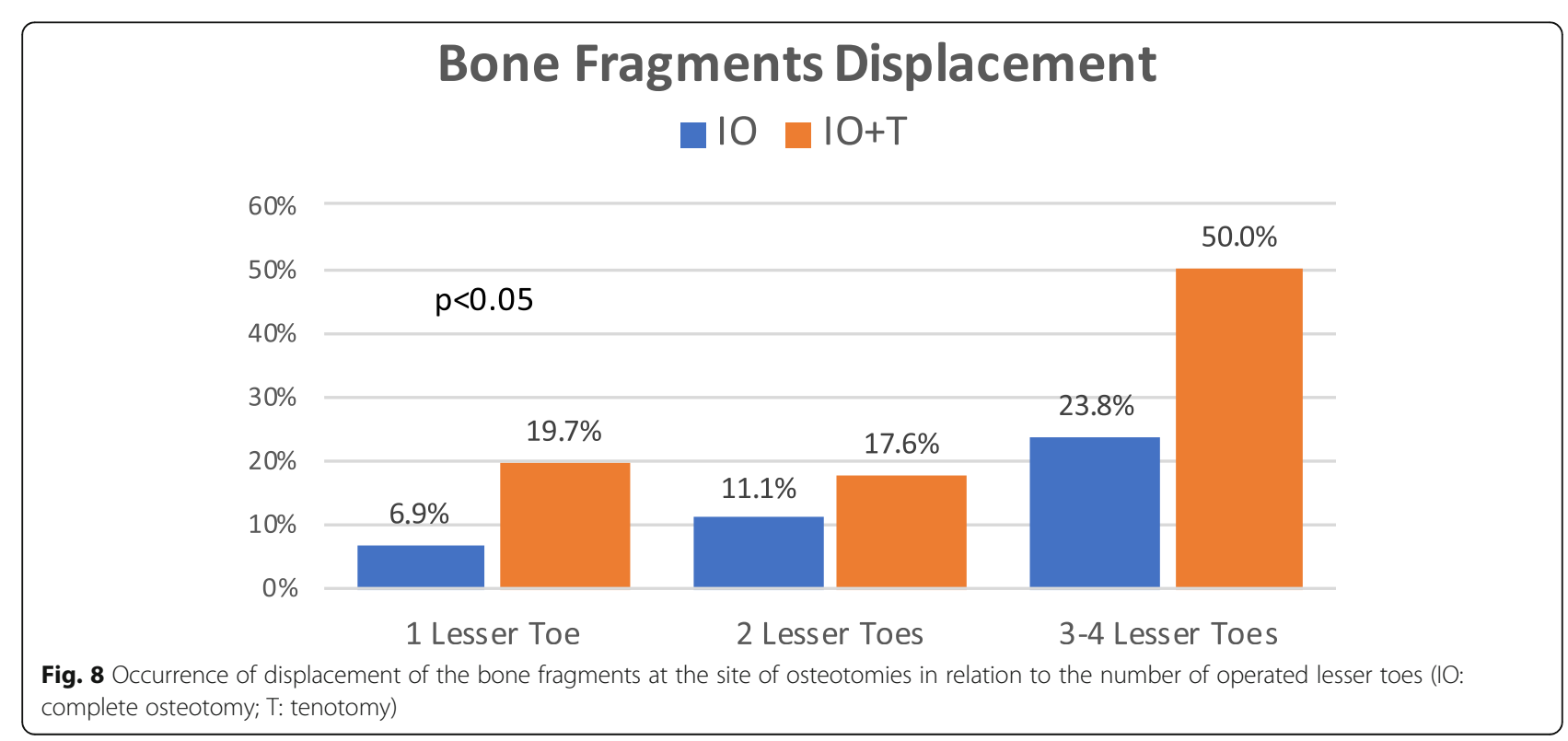




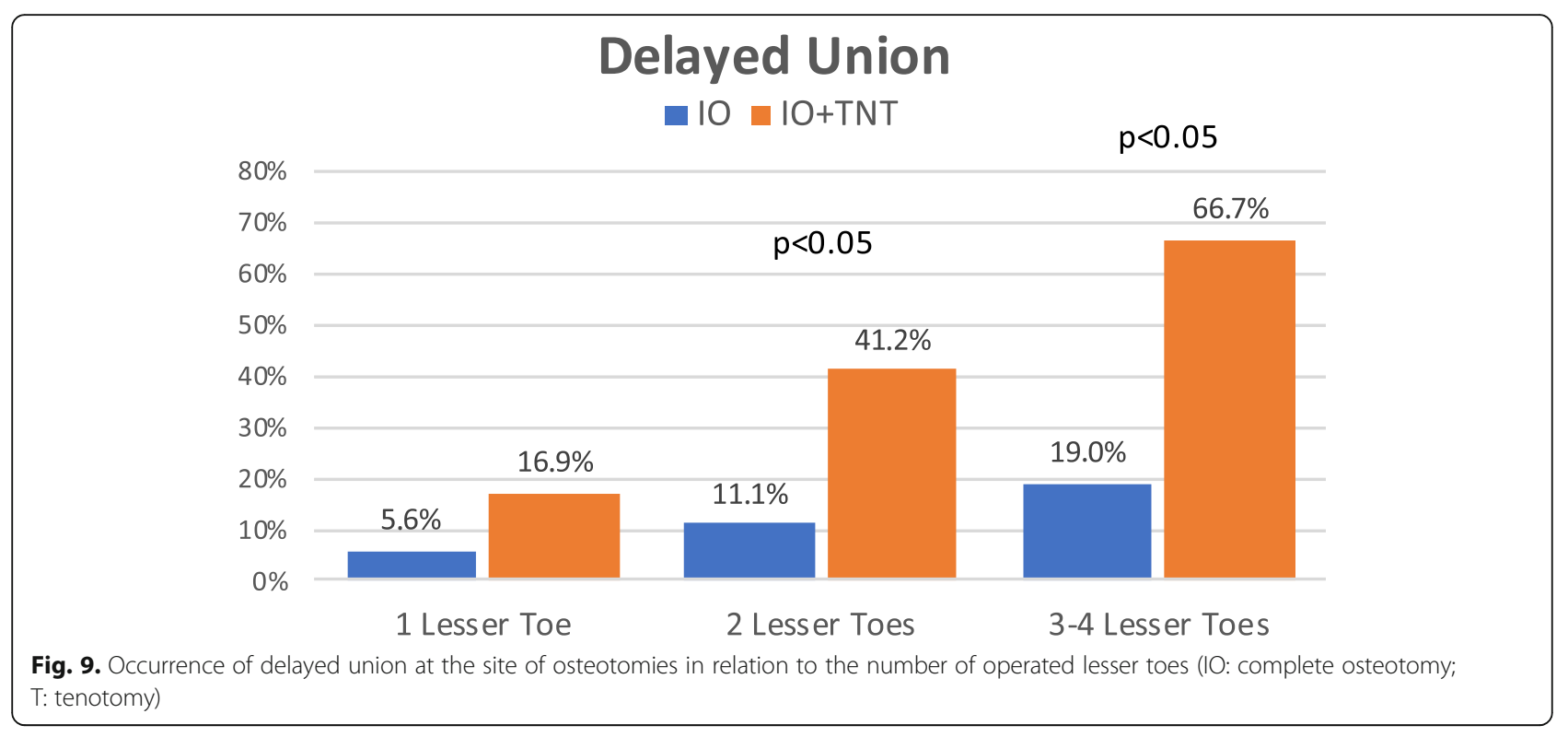

deformities [21, 22], others perform unicortical osteotomies in certain pathologies [16, 23], but all of them follow the same protocol starting correction with soft tissue techniques.

Our proposal to treat LTD using IO techniques reverses the order of execution performing first the osteotomies in the bone segments, from proximal to distal. The procedures on soft tissues are finally carried out if the deformity requires additional actions in case of residual deformities. Recently, the European Federation of National Associations of Orthopaedics and Traumatology (EFORT) also suggests the addressing of proximal deformities prior to distal deformities [4, 9]. According to our practice, once the IOs are performed, the degree of correction and equilibrium achieved between the intrinsic and extrinsic muscle groups is intra-surgically assessed, then deciding whether or not to perform the flexor and/or extensor tenotomies.

The results obtained with the application of IO techniques (723 osteotomies in 556 toes) show a highly relevant functional improvement according to AOFAS scores. The current study addresses for the first time the clinical benefit of partial or incomplete phalangeal osteotomies by a commonly accepted measurement scale. AOFAS scores at 1-year follow-up show the clinical efficacy of IO technique using MIS for LTD. Outcomes are comparable to other studies performing digital arthrodesis and arthroplasty with open techniques $[6-10,16]$. Although the AOFAS subscale focusing interphalangeal deformities of the lesser toes was only considered in this study, the results should be interpreted with caution. Some concerns have been raised with regard to AOFAS validity and reliability, but many clinicians continue to administer the AOFAS survey to patients. In fact, AOFAS scales continue to be some of the most widely used instruments in clinical studies, and they remain in use at a substantially higher rate than other scales that have been validated [23]. A relevant finding in our study is that the preoperative impairment of the AOFAS scores was dependent of the number of toes requiring surgical correction. Preoperative AOFAS scores decreased as the number of associated LTD increased. The clinical significance of these findings deserves further studies.

Most notably, the current study shows that the addition of flexor/extensor tenotomies to IO is also associated with good functional results, but worse than that found in cases without tenotomy. This finding is also confirmed by the earlier surgical discharge or the start of daily life activities in cases operated on by IO without tenotomies. Furthermore, the rate of adverse events was significantly higher when tenotomies were added to IO. Interestingly, in accordance with other MIS studies, no infection was found in the current series [24].

A commonly performed soft tissue MIS procedure in the lesser toes is a percutaneous flexor tenotomy. Debarge et al. [25] performed a percutaneous flexor tenotomy on 50 patients with clawing of the lesser toes secondary to shortening metatarsal scarf osteotomy. They found a $10 \%$ recurrence rate, and $4 \%$ of the patients developed complex regional pain syndrome. In cases with the same forefoot surgery but without flexor tenotomy, the rate of a toe grasping defect was significantly lower than in cases undergoing flexor tenotomy ( $4.3 \%$ versus $10 \%)$

In the current series, the addition of a flexor/extensor tenotomy triggers some of the adverse events, particularly the percentage of delayed union, hypertrophic callus, bony fragments displacement, postsurgical phalangeal fracture, and final malunion. Previous studies indicate that some of these 
adverse events can influence outcome after lesser toe surgery [26]. On the contrary, most of the Dutch orthopedic surgeons $(65 \%)$ stated that, when attempting interphalangeal arthrodesis, malunion and pseudarthrosis do not influence postoperative outcome [1]. Other authors showed that fusion was not required for a successful result following arthrodesis in cases with hammer toe [27].

In the present study, $57.8 \%$ of the operated toes by IO did not require additional tenotomies. This fact indicates that the alignment of the bone segments obtained with the osteotomies was able to reestablish the harmonic balance between the agonist and antagonist muscle groups, responsible for the digital mechanics, without the need to act on tendons. The new distribution of the force vectors on the bone surfaces could create an adequate compression and stability of the bone fragments. The design of the osteotomies preserving part of the cortical wall could help to reduce complications [28], providing a satisfactory functional recovery with a shorten postoperative period. The principal limitation of MIS to achieve satisfactory results is the way to perform and monitor the postoperative dressings that maintain toe alignment.

Diabetes mellitus, rheumatic diseases, and smoking seem to predispose to higher complication rates [26, 28]. As in the Gilheany et al. series [24], comorbidities did not translate to increased complication rates in the current cohorts. Similarly, none of our patients required an intraoperative conversion to open repair. Finally, the inclusion of patients of advanced age (over the age of 75) did not negatively influence both complication rate and outcome.

The stability provided by the cortical continuity when performing IO and the appropriate external bandages, responsible for closing the inter-fragmentary gap, maintains the contact between the bony surfaces. As IO preserves the articular surfaces, they are particularly indicated in reducible or semi-reducible digital deformities. In cases with joint dislocation or a deformity that requires shortening of the digit, IO combined with other MIS techniques such as complete osteotomies, toe arthroplasty, or arthrodesis would be desirable.

\section{Conclusion}

Despite the limitations inherent to the variety of LTD, this study shows the clinical efficacy of the partial or incomplete osteotomies performed by MIS starting from proximal to distal phalanx in cases with reducible deformity of the proximal interphalangeal joint. Combined to the HAV correction, IO provides relevant functional improvement according to AOFAS functional scores. IO provides a satisfactory correction of LTD with functional and biomechanical restoration, and a low percentage of complications. These percutaneous techniques should be considered as an alternative to the conventional surgery of lesser toe deformities. Notably, the addition of flexor/extensor tenotomies to IO enhances the occurrence of adverse events particularly delayed union, hypertrophic callus, and postoperative phalangeal fracture. The avoidance of tenotomies supposes a lesser surgical aggression and a lower physiological response to the surgical trauma, achieving an earlier functional recovery. Further studies investigating the effectiveness of IO techniques are needed particularly focusing on long-term outcomes, as well developing treatment algorithms to guide clinical decision making.

\begin{abstract}
Abbreviations
AOFAS: American Orthopaedic Foot and Ankle Society; HVA: Hallux abductus valgus; IO: Incomplete osteotomy; LTD: Lesser toe deformities; MIS: Minimally invasive surgery
\end{abstract}

\section{Acknowledgements}

Not applicable

\section{Authors' contributions}

ENGa performed the surgeries and analyzed and interpreted the data. JFT contributed to the study concept and design and participated in the figure designs. LRA and ENGz carried out the data collection and analysis. AMN contributed to the study concept and design and the interpretation of data. CB participated in the study design and the statistical analysis and was a major contributor in writing the manuscript. All authors read, discuss, and approved the final manuscript. The authors declare that this article represents honest work.

\section{Funding}

None

Availability of data and materials

The datasets used and/or analyzed during the current study are available from the corresponding author on reasonable request.

\section{Ethics approval and consent to participate}

Ethical approval was obtained from the Ethics in Research Committee of the University of Extremadura (ref. 102/2007). This clinical practice observational study was performed in accordance with the ethical standards of the 1964 Declaration of Helsinki as revised in 2000 and those of Good Clinical Practice. All patients received a thorough explanation of this study, and informed consent to participate was obtained from the participants.

\section{Consent for publication}

The patients gave their oral and written informed consent to the publication of their anonymous and clustered data and anonymous pictures.

\section{Competing interests}

The authors declare that they have no competing interests.

\section{Author details}

'Doctorate School, Valencia Catholic University San Vicente Martir, Valencia, Spain. ${ }^{2}$ School of Physiotherapy and Podiatry, Valencia Catholic University, Ramiro Maeztu 14, 46900 Torrent, Valencia, Spain. ${ }^{3}$ Podiatric School, University of Extremadura, Plasencia, Cáceres, Spain. ${ }^{4}$ Institute for Research on Musculoskeletal Disorders, Valencia Catholic University, Valencia, Spain.

Received: 22 June 2019 Accepted: 29 August 2019

Published online: 11 September 2019

\section{References}

1. Schrier JC, Louwerens JW, Verheyen CC. Opinions on lesser toe deformities among Dutch orthopaedic departments. Foot Ankle Int. 2007;28:1265-70.

2. Shirzad K, Kiesau CD, DeOrio JK, Parekh SG. Lesser toe deformities. J Am Acad Orthop Surg. 2011;1:9505-14.

3. Coughlin MJ. Lesser toe deformities. In: Coughlin MJSC, Anderson RB, eds. Mann's surgery of the foot and ankle. Ninth ed. Elsevier; 2014:322-424. 
4. Malhotra K, Davda K, Singh D. The pathology and management of lesser toe deformities. EFORT Open Rev. 2016;1:409-19.

5. Saro C, Bengtsson AS, Lindgren U, et al. Surgical treatment of hallux valgus and forefoot deformities in Sweden: a population-based study. Foot Ankle Int. 2008;29:298-304.

6. Fernández $\mathrm{CS}$, Wagner $\mathrm{E}$, Ortiz C. Lesser toes proximal interphalangeal joint fusion in rigid claw toes. Foot and Ankle Clinics. 2012;17(3):473-80.

7. Unsdorfer GL, Unsdorfer KML. Proximal phalangeal osteotomy with proximal interphalangeal joint arthrodesis for multiplanar deformities of the second toe: Historical Perspectives and Review of a Case Series. J Foot and Ankle Surg. 2011;50(6):687-94.

8. Kernbach KJ. Hammertoe surgery: arthroplasty, arthrodesis or plantar plate repair? Clin Podiatr Med Surg. 2012;29(3):355-66.

9. Louwerens JWSJ. Lesser toe deformities. In: Bentley G, editor. European surgical orthopaedics and traumatology. The EFORT textbook, vol. 6. Berlin Heidelberg: Springer-Verlag; 2014. p. 3407-973.

10. D'Angelantonio AM, Nelson-Rinaldi KA, Barnard J, Oware F. Master techniques in digital arthrodesis. Clin Podiat Med Surg. 2012;29(1):21-40.

11. Bauer T. Percutaneous forefoot surgery. Orthop Traumatol Surg Res. 2014; 100(1):S191-204

12. Biz C, Fosser M, Dalmau-Pastor M, Corradin M, Rodà MG, Aldegheri R, Ruggieri P. Functional and radiographic outcomes of hallux valgus correction by mini-invasive surgery with Reverdin-Isham and Akin percutaneous osteotomies: a longitudinal prospective study with a 48month follow-up. J Orthop Surg Res. 2016;11:157.

13. Boberg JS, Menn JJ, Brown WL. The distal Akin osteotomy: a new approach. J Foot Surg. 1991;30(5):431-6.

14. Sanhudo JV. Clinical tip: modified Akin osteotomy. Foot Ankle Int. 2005;26:901-2.

15. Redfern D, Vernois J, Legré BP. Percutaneous surgery of the forefoot. Clin Podiatr Med Surg. 2015;32(3):291-332.

16. Redfern $D$, Vernois J. Percutaneous surgery for metatarsalgia and the lesser toes. Foot Ankle Clin. 2016;21(3):527-50.

17. Frey-Ollivier $S$, Catena F, Hélix-Giordanino M, Piclet-Legré B. Treatment of flexible lesser toe deformities. Foot Ankle Clin. 2018;23(1):69-90.

18. Kitaoka HB, Alexander IJ, Adelaar RS, Nunley JA, Myerson MS, Sanders M. Clinical rating systems for the ankle-hindfoot, midfoot, hallux, and lesser toes. Foot Ankle Int. 1994;15(7):349-53.

19. Frey S, Hélix-Giordanino M, Piclet-Legré B. Percutaneous correction of second toe proximal deformity: proximal interphalangeal release, flexor digitorum brevis tenotomy and proximal phalanx osteotomy. Orthop Traumatol Surg Res. 2015;101(6):753-8.

20. Isham SA, Nunez OE. Isham Hammertoe procedures for the correction of lesser digital deformities. In: Maffulli N, Easley M, editors. Minimally Invasive Surgery of the Foot and Ankle. London: Springer; 2011. p. 171-83.

21. Maffulli N, Easley ME. Minimally invasive forefoot surgery in clinical practice [Internet]. Maffuli N, Easley M, editors. London: Springer; 2013 [cited 2017 Apr 25]. 235

22. Cazeau C, Doursounian L, Groupe de recherche et d'étude en chirurgie mini-invasive du pied et de la cheville. Chirurgie mini-invasive et percutanée du pied [Internet]. Sauramps médical; 2009

23. Pena F, Agel J, Coetzee JC. Comparison of the MFA to the AOFAS outcome tool in a population undergoing total ankle replacement. Foot Ankle Int. 2007;28(7):788-93.

24. Gilheany M, Omar Baarini O, Samaras D. Minimally invasive surgery for pedal digital deformity: an audit of complications using national benchmark indicators. J Foot Ankle Res. 2015;8:17.

25. Debarge R, Philippot R, Viola J, Besse JL. Clinical outcome after percutaneous flexor tenotomy in forefoot surgery. Int Orthop. 2009; 33(5):1279-82.

26. Femino JE, Mueller K. Complications of lesser toe surgery. Clin Orthop Rel Res. 2001:391:72-88.

27. Newman RJ, Fitton JM. An evaluation of operative procedures in the treatment of hammer toe. Acta Orthop Scand. 1979;50:709-12.

28. De Prado M. Complications in minimally invasive foot surgery. Fuß Sprunggelenk. 2013;11(2):83-94.

\section{Publisher's Note}

Springer Nature remains neutral with regard to jurisdictional claims in published maps and institutional affiliations.

\section{Ready to submit your research? Choose BMC and benefit from}

- fast, convenient online submission

- thorough peer review by experienced researchers in your field

- rapid publication on acceptance

- support for research data, including large and complex data types

- gold Open Access which fosters wider collaboration and increased citations

- maximum visibility for your research: over $100 \mathrm{M}$ website views per year

At BMC, research is always in progress.

Learn more biomedcentral.com/submissions 